\title{
Serum Uric Acid and Ambulatory Blood Pressure in Children With Primary Hypertension
}

\author{
DEBORAH P. JONES, PHYLLIS A. RICHEY, BRUCE S. ALPERT, AND RONGLING LI
}

Departments of Pediatrics [D.P.J., P.A.R., B.S.A.], Preventive Medicine [P.A.R., R.L.], University of Tennessee Health Science Center, Memphis, Tennessee 38103

\begin{abstract}
Hyperuricemia is associated with primary hypertension (HTN) in adults and children. Furthermore, uric acid levels during childhood are associated with blood pressure (BP) levels in adulthood. We measured 24-h ambulatory BP and serum uric acid (SUA) in 104 children referred for possible hypertension. Mean age was $13.7 \pm 2.6$ y (range, 7-18 y) with 67 males and 37 females; 74 were African-American, 29 Caucasian and one Asian. SUA was associated with age $(r=0.38, p=0.0001)$ and BMI Z-score $(r=$ $0.23, p=0.021)$. SUA was significantly associated with mean ambulatory systolic (S) and diastolic (D) BP. Mean ambulatory BP was normalized to gender- and height-specific reference standards using BP index. SUA was significantly associated with 24-h DBP index and nocturnal DBP index after adjusting for age, gender, race, BMI Z-score and urinary sodium excretion. SUA was also significantly associated with 24-h DBP load and nocturnal DBP load. Uric acid was significantly associated with increased likelihood for diastolic HTN (OR $=2.1, \mathrm{CI}=1.2,3.7 ; p=0.0063)$ after adjusting for other co-variables. Among children at risk for HTN, the likelihood for diastolic HTN (as defined by ambulatory blood pressure monitoring) increases significantly as SUA increases. SUA may be associated with increased severity of HTN during youth. (Pediatr Res 64: 556-561, 2008)
\end{abstract}

$S^{\prime}$ tudies in adults indicate that uric acid is an independent risk factor for cardiovascular disease (1). Elevated serum uric acid (SUA) levels are frequently seen in adults with hypertension (HTN); this phenomenon was initially thought to reflect subtle renal damage known to accompany established HTN. However, uric acid has been hypothesized to play a role in the early stages of vascular damage and contribute to the development of HTN (2). Hyperuricemia is associated with increased risk for development of HTN in adults (3-5).

Among participants in the Bogalusa Heart Study, SUA levels measured during childhood were significantly associated with childhood and adult systolic blood pressure (SBP) and diastolic blood pressure (DBP) (6). In addition, the change in SUA from youth to adulthood was a significant predictor of adult SBP. In children with HTN (defined by increased casual BP), an SUA level of $>5.5 \mathrm{mg} / \mathrm{dL}$ was significantly associated with the occurrence of primary HTN; casual SBP and DBP

Received April 4, 2008; accepted June 4, 2008.

Correspondence: Deborah P. Jones, M.D., Department of Pediatrics, University of Tennessee Health Science Center, 50 North Dunlap, Room 301, Memphis, TN 38103; e-mail: dpjones@utmem.edu

Supported by a grant from the Children's Foundation Research Center of Memphis at Le Bonheur Children's Medical Center, and the UTHSC GCRC NCRR \#000211 and NIH NHLBI \#5K23HL83910-2. were significantly correlated with SUA (7). Unfortunately, neither of the above studies adjusted casual BP levels in their pediatric subjects for gender, age and height related normal values. In addition, HTN in the second study was defined based upon casual BP values.

Ambulatory blood pressure monitoring (ABPM) is superior to casual clinic measurement in the assessment of BP and cardiovascular risk. In adults with hypertension (HTN), ABPM has consistently demonstrated superiority over casual clinic readings for prediction of cardiovascular morbidity and mortality (8-10). Compared with office BP readings, ambulatory $\mathrm{BP}(\mathrm{ABP})$ is a better predictor of increased left ventricular mass in children with HTN $(11,12)$. To the best of our knowledge, studies investigating the relationship of ABP and SUA levels have not been published. The relationship between SUA and ABP was examined using a cross-sectional study design in a multiethnic cohort of children who were referred for possible HTN.

\section{METHODS}

Children ages 6-18 y with a casual SBP or DBP $\geq 90$ th percentile were eligible to participate in the study (13). Subjects were excluded if they had received anti-hypertensive therapy, were on medications associated with HTN, or had known renal or cardiac disease. The majority of subjects were recruited from referrals to the UTMG subspecialty clinics after the finding of an elevated casual BP in the primary care office, however, they had not consistently had increased BP levels on three separate occasion before entry into the study. Informed consent was obtained; the research protocol was approved by the University of Tennessee Health Science Center Institutional Review Board and followed the guidelines for good clinical practice. Subjects found to have HTN underwent additional studies based upon the current guidelines (14) to ascertain whether they had a secondary cause for HTN. Five children were found to have secondary HTN and are not included in this analysis.

ABPM was performed using the AM5600 ambulatory blood pressure monitor (Advanced Biosensor, Columbia, SC) (15). The monitors were programmed to measure the BP every 20 min for a 24 -h period using the auscultatory technique (with oscillometric "back-up" technique) to detect SBP at Korotkoff phase I and DBP at Korotkoff phase V. The appropriate sized cuff was chosen according to the recommendations of the Working Group with the width of the bladder at least $40 \%$ of the mid-arm circumference (13). The brachial artery was located at the anticubital fossa of the nondominant arm, and the microphone was taped to the subject's arm over the strongest impulse, followed by placement of the cuff and electrodes (15). During the AM5600 "office check period," with the subject in a seated position, a minimum of three readings were taken simultaneously in the same

Abbreviations: ABP, ambulatory blood pressure; ABPM, ambulatory blood pressure monitoring; BP, blood pressure; BPI, blood pressure index; DBP, diastolic blood pressure; HTN, hypertension; SBP, systolic blood pressure; SUA, serum uric acid; $\mathbf{U ~} \mathbf{N a} / \mathbf{c r}$, urinary sodium to creatinine ratio 
arm via a three-way stopcock, by auscultation with a mercury sphygmomanometer and stethoscope, and the AM5600, using the recorder's BP cuff. The calibration readings allowed for adjustments to be made in the recorder's microphone amplification and to establish a baseline for ABPM versus manual SBP and DBP. The office check readings were not included in the 24-h results. Each subject's parent/guardian was asked to record the subject's bedtime and time of awakening. After $24 \mathrm{~h}$, the cuff and monitor were removed, and the data downloaded using the manufacturer's software (12).

Subject height and weight were measured using a balance beam scale and pediatric wall-mounted stadiometer. Height percentile was calculated using the CDC NHANES III data tables by age in months (16). Body mass index (BMI) was calculated as weight $(\mathrm{kg})$ divided by the square of height $\left(\mathrm{m}^{2}\right)$. BMI Z-scores, which reflect the SD score for the age- and gender-appropriate BMI distribution, were calculated using the same methods as used in the 2000 CDC Growth Charts for the United States (16).

The mean SBP and DBP were calculated separately for the 24-h period and for awake and asleep periods. The ambulatory BP index (BPI) for each subject was calculated by expressing the mean $\mathrm{BP}$ as a ratio to the appropriate 95 th percentile for ambulatory BP (17). When the BPI is greater than 1.0, the mean BP is above the 95th percentile. HTN is defined as BPI $>1$. White coat HTN is defined as a casual BPI $>1$ but ABPM BPI $<1$. Masked HTN is defined as a casual BPI $<1$ but ABPM daytime BPI $>1$. BP load was calculated as the number of readings over the 95th percentile within each time period and was expressed as a percent of total readings for the period (7). Mean casual BP and casual BP index were calculated from the three readings obtained during the calibration period using a mercury sphygmomanometer. Casual BP index was calculated as the ratio of the mean casual BP over the 95th percentile (13).

Blood samples for the measurement of SUA and metabolic profile were collected before placing the ambulatory monitor. A 24-h urine sample was collected to measure urinary excretion of sodium and creatinine. To correct for any missed samples in the timed urine collection, 24-h urinary $\mathrm{Na}$ excretion ( $\mathrm{mmol} / 24-\mathrm{h})$ was standardized to urinary creatinine excretion $(\mathrm{g} /$ 24-h), expressed as urinary Na:creatinine ratio $\left(\mathrm{U} \mathrm{Na}^{+} / \mathrm{cr}\right)$. Creatinine clearance was estimated using the Schwartz equation, which uses height and serum creatinine to estimate GFR expressed as $\mathrm{ml} / \mathrm{min} / 1.73 \mathrm{~m}^{2}$ (18).

Statistical analyses. SAS 9.1 (SAS, Inc., Cary, NC) statistical software was used for all analyses. Descriptive analyses were used to assess the mean values and SD of means. Pearson correlation coefficients were calculated for all continuous measurements. Effect of SUA on ABP was assessed by linear regression. Logistic regression analysis was performed using the presence of systolic or diastolic hypertension as the dichotomous dependent variable and SUA as the continuous, independent variable. Multivariable analysis was performed to control for age, race and gender, as well as BMI Z-score and/or $\mathrm{U} \mathrm{Na} /$ cr. Since there are numerous definitions of normal uric acid levels during childhood, subjects were classified according to SUA using the recently published value of $\leq 4.9 \mathrm{mg} / \mathrm{dL}$ for the cut-point for analysis (19). After dichotomizations according to SUA, the likelihood for increased SUA was assessed using logistic regression; we controlled for the other covariables in this analysis.

\section{RESULTS}

Ambulatory BP and SUA data were successfully collected from 104 children. Of the subjects, 74 were African-American (AA), 29 were European American (EA) and one was Asian; 37 were female $(\mathrm{F})$ and 67 male $(\mathrm{M})$. The mean age was $13.7 \pm 2.6 \mathrm{y}$ with a range of $7-18 \mathrm{y}$. Mean BMI Z-score was $1.49 \pm 1.02$ with a range of $-1.47-3.08$. Fifty-five subjects had a BMI percentile that classified them as overweight. The mean SUA for all subjects was $4.91 \pm 1.3$ with a range of 2.5-9.1 mg per deciliter. Males had significantly higher SUA levels than females $(5.36 \pm 1.32$ versus $4.08 \pm 0.76 \mathrm{mg} / \mathrm{dL}$, $p<0.0001)$ and African-Americans had significantly lower SUA levels compared with Caucasian subjects $(4.69 \pm 1.25$ versus $5.46 \pm 1.31 \mathrm{mg} / \mathrm{dL}, p=0.0016)$. Characteristics of the participants are summarized by gender and race in Table 1.

Based upon casual blood pressure index, $54 \%$ of subjects were classified as having systolic HTN and $16 \%$ were classified as having diastolic HTN. According to the $24 \mathrm{~h} \mathrm{ABP}$ index, $39 \%$ of subjects had systolic HTN and $21 \%$ had diastolic HTN. Twenty-three percent and $6 \%$ were classified as having white coat systolic and diastolic HTN, respectively (white coat HTN was defined as a casual BP $\geq 95$ th and a mean $24 \mathrm{~h} \mathrm{ABP}<95$ th percentile); $7 \%$ and $4 \%$ were classified as having masked systolic and diastolic HTN, respectively (Masked HTN was defined as a casual BP $<95$ th percentile and a mean daytime ABP $>95$ th percentile). Sixty-three percent of subjects had an SBP load greater than $25 \%$ and $38 \%$ had a DBP load greater than $25 \%$.

Mean SUA levels were significantly higher among individuals with diastolic HTN as defined as a 24-h DBPI >1.0: $5.55 \pm 1.73 \mathrm{mg} / \mathrm{dL}$ versus $4.75 \pm 1.12 \mathrm{mg} / \mathrm{dL}, p=0.0077$. There were no significant differences in mean SUA between subjects with and without systolic HTN-either defined by 24-h SBPI or SBP load. Subjects whose SUA was $>4.9$ $\mathrm{mg} / \mathrm{dL}$ had an OR of $2.09(\mathrm{CI}=1.07,4.09, p=0.022)$ for increased 24-h DBPI after controlling for race, gender, age and BMI Z-score.

SUA was significantly associated with casual SBP $(r=$ $0.32, p=0.0012)$ and DBP $(r=0.23, p=0.025)$. However, after transformation of the casual BP into a BPI, the correlation was no longer significant. SUA was significantly positively correlated with BMI Z-score $(r=0.23, p=0.021)$ and age $(r=0.37, p=0.0002)$; SUA was negatively associated with $\mathrm{U} \mathrm{Na}^{+} / \mathrm{cr}(r=-0.255, p=0.126)$. Stratification based upon race revealed that SUA was significantly correlated with $\mathrm{U} \mathrm{Na}^{+} / \mathrm{cr}$ in African-Americans $(r=-0.314, p=0.0085)$, but not significantly correlated to $\mathrm{U} \mathrm{Na}^{+} / \mathrm{cr}$ in Caucasians $(r=$ $-0.236, p=0.255)$. SUA was not significantly correlated with estimated creatinine clearance.

The relationship of SUA with ABP parameters is shown in Table 2. Because of the known gender, age and ethnic differences in SUA, multivariable linear regression analysis was performed to control for gender, age and race. After controlling for age, gender and race, both SBP and DBP (except daytime DBP) continued to show significant association with SUA. After adding BMI Z-score, 24-h and nighttime SBP and DBP continued to demonstrate a significant relationship with SUA. There was a significant relationship between SUA and 24-h DBPI that was strengthened after controlling for demographic variables. This relationship was also significant after adding BMI Z-score. There was a significant relationship between SUA and 24-h DBP load, which was strengthened after controlling for demographic variables; this relationship was also significant after adding BMI Z-score to the other variables. Serum uric acid is plotted according to mean $24 \mathrm{~h}$ DBP and $24 \mathrm{~h}$ DBPI in Figure 1.

Urinary $\mathrm{Na}^{+}$excretion may have an impact on uric acid excretion, and therefore on SUA (20). Since we found a significant relationship between SUA and $\mathrm{U} \mathrm{Na}^{+} / \mathrm{cr}$, we also added $\mathrm{U} \mathrm{Na}^{+} / \mathrm{cr}$ to the multivariable linear regression analysis (age, gender, race, and BMI Z-score). Twenty-four-hour DBPI, nighttime DBPI, 24-h DBP load, and nighttime DBP load continued to demonstrate a significant relationship with SUA with all variables in the model.

The odds ratio (OR) was 1.59 (95\% CI: $1.09-2.31, p=$ 0.0152) for diastolic HTN associated with each $1 \mathrm{mg}$ per deciliter increase of SUA. After adjusting for age, race and 
Table 1. Characteristics of study participants

\begin{tabular}{|c|c|c|c|c|}
\hline & $\begin{array}{l}\text { AA males } \\
(n=44)\end{array}$ & $\begin{array}{l}\text { AA females } \\
\quad(n=30)\end{array}$ & $\begin{array}{l}\text { EA males } \\
(n=22)\end{array}$ & $\begin{array}{l}\text { EA females } \\
\quad(n=7)\end{array}$ \\
\hline Age (y) & $14.0 \pm 0.4$ & $12.9 \pm 0.5$ & $13.9 \pm 0.5$ & $13.3 \pm 1.0$ \\
\hline BMI z-score & $1.6 \pm 0.1$ & $1.4 \pm 0.2$ & $1.7 \pm 0.22$ & $1.4 \pm 0.4$ \\
\hline SUA (mg/dL) & $5.07 \pm 0.2$ & $4.09 \pm 0.2 *$ & $5.94 \pm 0.2 \dagger$ & $4.01 \pm 0.4^{*}$ \\
\hline Urine $\mathrm{Na} / \mathrm{cr}$ & $105.1 \pm 7.2$ & $117.1 \pm 9.2$ & $117.2 \pm 13$ & $112.0 \pm 18$ \\
\hline Casual SBP (mm Hg) & $131.9 \pm 1.7$ & $125.9 \pm 2.2^{*}$ & $132.9 \pm 2.7$ & $128.4 \pm 7.8$ \\
\hline Casual DBP (mm Hg & $73.9 \pm 1.1$ & $73.7 \pm 1.6$ & $76.4 \pm 1.8$ & $75.6 \pm 2.7$ \\
\hline 24-h SBP (mm Hg) & $126.6 \pm 1.3$ & $117.9 \pm 1.5^{*}$ & $126.2 \pm 1.7$ & $121.8 \pm 6.9$ \\
\hline 24-h DBP (mm Hg) & $71.6 \pm 0.9$ & $69.3 \pm 1.3$ & $72.2 \pm 1.3$ & $72.8 \pm 3.8$ \\
\hline Day SBP (mm Hg) & $131.9 \pm 1.3$ & $122.7 \pm 1.6^{*}$ & $132.82 \pm 1.9$ & $127.2 \pm 6.8$ \\
\hline Day DBP (mm Hg) & $75.4 \pm 1.1$ & $73.2 \pm 1.4$ & $76.86 \pm 1.37$ & $78.6 \pm 3.6$ \\
\hline Night SBP (mm Hg) & $118.9 \pm 1.6$ & $112.5 \pm 1.8^{*}$ & $117.09 \pm 1.93$ & $114.7 \pm 7.7$ \\
\hline Night DBP (mm Hg) & $66.4 \pm 1.2$ & $64.8 \pm 1.6$ & $65.67 \pm 1.39$ & $64.3 \pm 4.1$ \\
\hline 24-h SBPI $\$$ & $0.99 \pm 0.01$ & $0.96 \pm 0.01 *$ & $0.99 \pm 0.01$ & $1.01 \pm 0.06$ \\
\hline 24-h DBPI & $0.93 \pm 0.01$ & $0.91 \pm 0.02$ & $0.94 \pm 0.02$ & $0.97 \pm 0.06$ \\
\hline Day SBPI & $0.99 \pm 0.01$ & $0.95 \pm 0.01 *$ & $0.99 \pm 0.02$ & $1.00 \pm 0.05$ \\
\hline Day DBPI & $0.88 \pm 0.01$ & $0.87 \pm 0.02$ & $0.90 \pm 0.02$ & $0.95 \pm 0.05 \dagger$ \\
\hline Night SBPI & $1.01 \pm 0.01$ & $1.00 \pm 0.02$ & $0.99 \pm 0.02$ & $1.02 \pm 0.06$ \\
\hline Night DBPI & $0.99 \pm 0.02$ & $0.98 \pm 0.02$ & $0.98 \pm 0.02$ & $0.98 \pm 0.07$ \\
\hline Nocturnal dip SBP \% & $13.0 \pm 1.2$ & $10.2 \pm 1.2$ & $15.7 \pm 1.5$ & $12.5 \pm 3.2$ \\
\hline Nocturnal dip DBP \% & $9.1 \pm 1.2$ & $8.4 \pm 1.0$ & $11.2 \pm 1.3$ & $14.3 \pm 2.4 \dagger$ \\
\hline 24-h SBP load \% & $45 \pm 3$ & $36 \pm 4$ & $44 \pm 6$ & $49 \pm 15$ \\
\hline 24-h DBP load \% & $28 \pm 3$ & $26 \pm 4$ & $31 \pm 4$ & $44 \pm 12$ \\
\hline Day SBP load \% & $42 \pm 4$ & $28 \pm 4 *$ & $43 \pm 7$ & $50 \pm 15$ \\
\hline Day DBP load \% & $19 \pm 3$ & $15 \pm 4$ & $23 \pm 4$ & $38 \pm 11 \dagger$ \\
\hline Night SBP load \% & $46 \pm 5$ & $47 \pm 6$ & $39 \pm 7$ & $49 \pm 16$ \\
\hline Night DBP load \% & $42 \pm 5$ & $42 \pm 6$ & $38 \pm 6$ & $34 \pm 14$ \\
\hline
\end{tabular}

Day refers to readings obtained during the "awake" period and night refers to readings obtained during the "sleep" period.

* Gender difference comparison, $p<0.05$.

$\dagger$ Racial difference comparison, $p<0.05$.

$\$ \mathrm{BP}$ index is the subject's mean BP divided by the 95th percentile. Task force reference standards (13) were used for casual BP and ambulatory standards for ABP (17). A BP index $>1$ is the definition of HTN.

Table 2. Regression analysis of ambulatory BP and serum uric acid

\begin{tabular}{|c|c|c|c|c|c|c|}
\hline & \multicolumn{2}{|c|}{ Model $1 *$} & \multicolumn{2}{|c|}{ Model $2 \dagger$} & \multicolumn{2}{|c|}{ Model $3 \ddagger$} \\
\hline & $\beta$ & $p$ & $\beta$ & $p$ & $\beta$ & $p$ \\
\hline \multicolumn{7}{|l|}{ Systolic BP } \\
\hline Mean $24 \mathrm{~h}$ & 2.81 & 0.0003 & 2.19 & 0.02 & 1.77 & 0.048 \\
\hline Mean day & 2.59 & 0.0015 & 1.83 & NS & 1.47 & NS \\
\hline Mean night & 2.47 & 0.0041 & 2.60 & 0.015 & 2.15 & 0.043 \\
\hline BPI $24 \mathrm{~h}$ & 0.0122 & 0.0378 & 0.0171 & 0.015 & 0.0135 & 0.0561 \\
\hline BPI day & 0.0099 & NS & 0.0133 & NS & 0.0103 & NS \\
\hline BPI night & 0.0063 & NS & 0.0198 & 0.030 & 0.0149 & NS \\
\hline Load $24 \mathrm{~h}$ & 2.95 & NS & 6.66 & 0.01 & 5.41 & 0.0366 \\
\hline Load day & 3.33 & NS & 4.98 & 0.0497 & 3.774 & NS \\
\hline Load night & 2.09 & NS & 7.645 & 0.0152 & 5.647 & NS \\
\hline \multicolumn{7}{|l|}{ Diastolic BP } \\
\hline Mean $24 \mathrm{~h}$ & 1.60 & 0.0031 & 1.73 & 0.0112 & 1.55 & 0.0187 \\
\hline Mean day & 1.15 & NS & 1.27 & NS & 1.07 & NS \\
\hline Mean night & 1.77 & 0.0041 & 2.31 & 0.0031 & 2.21 & 0.0048 \\
\hline BPI $24 \mathrm{~h}$ & 0.0178 & 0.0094 & 0.0239 & 0.005 & 0.0219 & 0.0121 \\
\hline BPI day & 0.0113 & NS & 0.0163 & 0.0503 & 0.0142 & NS \\
\hline BPI night & 0.0226 & 0.0154 & 0.0354 & 0.0023 & 0.0343 & 0.0041 \\
\hline Load $24 \mathrm{~h}$ & 3.50 & 0.0543 & 6.58 & 0.0027 & 6.42 & 0.0044 \\
\hline Load day & 2.51 & NS & 3.87 & 0.0513 & 3.23 & NS \\
\hline Load night & 5.06 & 0.0327 & 8.54 & 0.0035 & 8.26 & 0.0062 \\
\hline
\end{tabular}

ABP parameters are 24-h, diurnal and nocturnal BP load, which expresses the percent of readings greater than the 95th percentile as a fraction of the entire number of readings for each period, not as a percent.

$\beta=$ parameter estimate for regression slope.

* Model 1 is the univariate analysis between SUA and ABP parameters.

$\dagger$ Model 2 is the multivariable analysis between SUA and ABP parameters while controlling for age, gender and race.

\$ Model 3 is the multivariable analysis between SUA and ABP parameters while controlling for age, gender, race and BMI Z-score. 

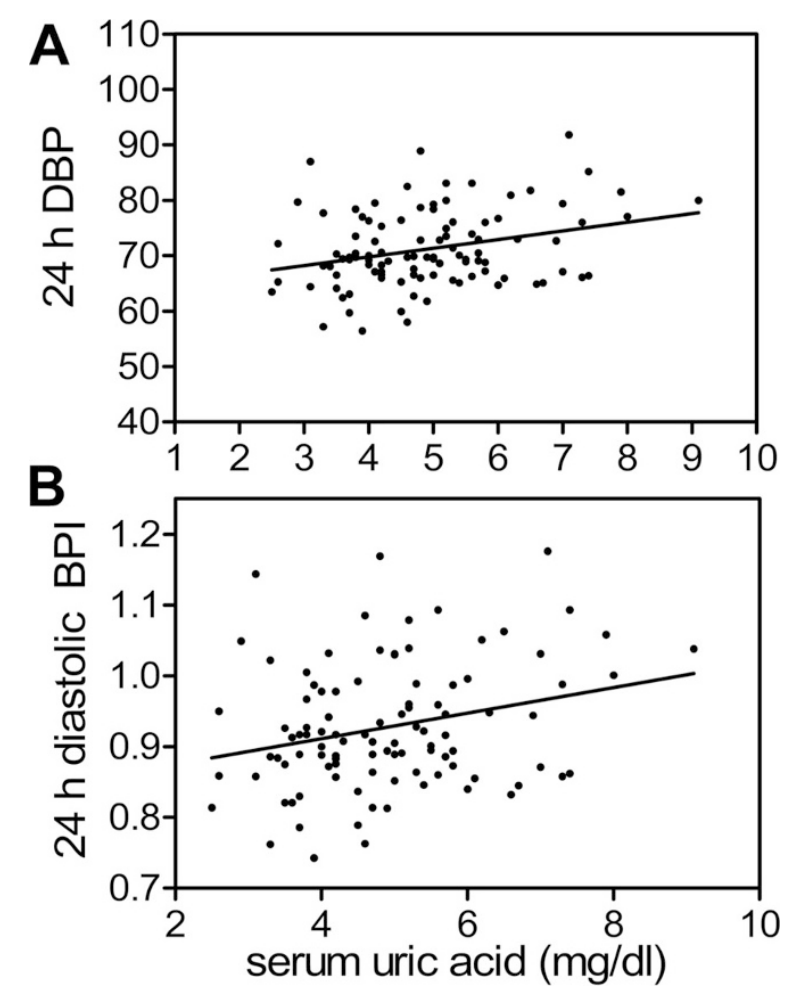

Figure 1. SUA plotted against mean $24 \mathrm{~h}$ diastolic BP $(A)$ and 24-h DBP index $(B)$. Pearson correlation coefficient for mean $24 \mathrm{~h} \mathrm{DBP}=0.29, p=$ 0.0033 , and for $24 \mathrm{~h}$ DBPI $=0.26, p=0.0094$.

gender the OR increased to 2.36 with $95 \%$ CI: $1.38-4.02(p=$ 0.0016). After addition of BMI Z-score, the OR was 2.14 (95\% CI: $1.25-3.66, p=0.0055)$ and remained unchanged after controlling for $\mathrm{U} \mathrm{Na} / \mathrm{cr}$. The OR for 24-h DBP load $>50 \%$ was 1.44 (95\% CI: $1.04-2.11, p=0.0308$ ) for each 1 $\mathrm{mg} / \mathrm{dL}$ increase in SUA; after controlling for age, race and gender, the OR was 2.41(95\% CI: $1.43-4.05, p=0.0009$ ). When the BMI Z-score was added into the model, the OR = 2.24 was slightly attenuated (95\% CI: $1.33-3.79, p=0.026)$. However, after addition of $\mathrm{U} \mathrm{Na} / \mathrm{cr}$, the OR was 2.04 (95\% CI: $1.15-3.62, p=0.0149)$.

There are several available references to normal values of SUA from infancy through adolescence; these differ somewhat in their definition of hyperuricemia. However, there appears to be a definite increase in the average serum uric acid after the age of $12 \mathrm{y}$. During adolescence, the gender related differences are consistently noted. We performed additional analysis to examine whether the relationship between SUA and ABP was different in the younger $(<12$ y of age, $n=25)$ and those $\geq 12$ y of age. There were no differences in our results when age was dichotomized in this manner. There was no interaction between preadolescent versus adolescent age in the relationship between SUA and ABP.

\section{DISCUSSION}

This is the first report to describe the relationship between ABP and SUA in children. SUA is significantly associated with 24-h DBP and nocturnal DBP. This relationship is strengthened after controlling for age, gender and race, and persists after controlling for BMI Z-score and urinary $\mathrm{Na}^{+}$ excretion. BMI is a confounder in the relationship between SUA and BP; this is not surprising, as numerous studies have demonstrated a significant effect of BMI on BP levels in childhood (21). The risk for diastolic HTN increases significantly as SUA increases. Since all except one subject with diastolic HTN also had systolic HTN, the increased risk for diastolic HTN found as the level of SUA increases may implicate uric acid as a marker for the severity of HTN in children. Previous studies in children neither consistently control for factors such as age, gender, and race nor they standardize BP levels for the known age, gender and height related differences (7).

The analysis of association between SUA and BP among youth is complicated by the observed developmental changes in SUA and urinary uric acid excretion. SUA increases with age and BMI during childhood. Gender and racial differences have also been observed $(22,23)$. Gender differences in the normal serum uric acid levels become most obvious during adolescence, and have been attributed to the effect of estrogen. We found no significant interaction of age group $<12$ y versus $\geq 12$ y on the relationship between SUA with ABP. Degree of ponderosity and prevalence of the metabolic syndrome are associated with hyperuricemia $(19,24)$. Although our study did not assess for presence of the metabolic syndrome, we did find a correlation of SUA with BMI and BMI Z-score, and we controlled for this in our analysis.

Age-, size- and gender-related changes in BP make the analysis of BP data from children of different ages challenging; these type of analyses are handled by representation of $\mathrm{BP}$ to reference standards $(13,14)$. The most commonly used parameter, the BPI, allows analysis of BP levels between children of variable age, gender and size in relationship to the 95th percentile (25). Studies that report an association between SUA and BP during childhood are limited because casual BP levels were used in the analysis without correction for the appropriate normal threshold values. Since both BP and SUA increase with age, their correlation may have been overestimated. We also found a significant relationship between SUA and both casual and ABP, however, when the BP was expressed as BPI to control for known differences in BP with age, gender and height, the correlation did not persist. It is possible that casual BPI is significantly associated with SUA if a larger sample size was available for analysis.

Among adults, the association between SUA and BP has been known for decades $(26,27)$. The finding that SUA predicts the development of HTN, independently of other known risk factors, has re-emphasized the potential role of SUA as a cardiovascular risk factor $(2,3)$. Hyperuricemia is also associated with endothelial dysfunction and progressive renal disease $(1,28)$. Potential mechanisms by which SUA induces HTN and renal vascular disease have been recently elucidated from the hyperuricemic animal model (2). Vascular smooth muscle cells transport uric acid into the cytosol, where it stimulates cellular proliferation (29). Rats with hyperuricemia develop salt-sensitive HTN, which is associated with increased thickness of the afferent arteriole. In these animals glomerular vascular alterations are accompanied by decreased renin and nitric oxide synthase activity of the juxtaglomerular 
apparatus and can be prevented by administration of compounds that reduce SUA (30). These studies support a role for uric acid in the induction of HTN and pathologic renal vascular alterations (31). Since these vascular changes are likely to occur relatively early in the course of essential HTN, the investigation of youth at risk for HTN is a plausible approach to help in the understanding the role of SUA in human HTN (31).

The increased prevalence of hyperuricemia among children with essential HTN was originally reported by Prebis et al. (20). The multiethnic cohort of 31 children displayed a prevalence of hyperuricemia of $42 \%$ while ingesting an unrestricted diet. Hyperuricemia was attributed to reduced renal excretion since the fractional excretion of UA was inversely related to SUA. These authors observed a significant change in SUA based upon dietary sodium, presumably due to the effect of sodium excretion on uric acid excretion. The relationship between BP level and SUA was not examined in this early report (20). In a subsequent study which analyzed data from 6768 children ages 12-17 y, supine SBP and DBP were significantly correlated with SUA (23). SUA predicted SBP after controlling for gender, age, race, height, weight, and sexual maturity (23). Feig and Johnson (7) reported a significant correlation between SUA and casual BP in normal children, as well as those with primary HTN, a relationship which remained significant after controlling for BMI percentile. In addition, an SUA $>5.5 \mathrm{mg} / \mathrm{dL}$ was significantly associated with increased likelihood to have primary, but not secondary, HTN (7). However, the ethnic composition of that cohort was not reported and the analysis did not control for age, gender or race. In participants in the Bogalusa Heart Study, SUA during childhood correlated with casual SBP and DBP both during childhood and adulthood (6). The change in SUA from childhood to adulthood (after adjusting for age at which sampled during childhood) was a significant predictor of adult BP (6).

Limitations of the current study include the choice of ABP reference standards. We elected to use the European reference standards, which were collected using an oscillometric device. The accuracy and precision of the oscillometric monitors, used by Soergel et al. (17), were confirmed by simultaneous measurements with a mercury sphygmomanometer. We also validated the AM5600 using a mercury manometer according to the rigorous standards of the AAMI and the British Society of Hypertension. Because both types of devices are checked for accuracy against the gold standard, it would seem that they should provide comparable measurements. In addition, the demographic composition of our clinical cohort is very different from the homogeneous composition of the European cohort. A recent review on clinical and research applications of ABPM discusses this very important issue (32). Because it is the largest normative data set collected thus far, the European normative data has become the standard for most clinicians and investigators using ABPM. Although not perfect, it may be the best choice at present. The last issue, which is the setting in which the data are collected-ambulatory versus seated-is possibly the most compelling rationale for using the European ambulatory data set. The clinical cohort in this study is not representative of the population of children in general, but is characteristic of children who are referred for evaluation for possible HTN.

In conclusion, among a multiethnic cohort of children referred for primary HTN, SUA was associated with ambulatory DBP after controlling for age, gender, race, BMI Z-score and urinary $\mathrm{Na}^{+}$excretion. Hyperuricemia among children with a strong family history of HTN or an elevated casual BP level is associated with more severe HTN and may be a marker of early cardiovascular damage. Measurement of SUA in children may prove to be a valuable tool in the assessment of cardiovascular risk as well as a target for risk-reduction therapy.

Acknowledgments. We thank the study participants and their families along with the dedicated study coordinators. The authors also thank the editorial assistance of Andrea Patters, B.S.

\section{REFERENCES}

1. Johnson RJ, Segal MS, Srinivas T, Ejaz A, Mu W, Roncal C, Sanchez-Lozada LG, Gersch M, Rodriguez-Iturbe B, Kang DH, Acosta JH 2005 Essential hypertension, progressive renal disease, and uric acid: a pathogenetic link? J Am Soc Nephrol 16:1909-1919

2. Johnson RJ, Kang DH, Feig D, Kivlighn S, Kanellis J, Watanabe S, Tuttle KR, Rodriguez-Iturbe B, Herrera-Acosta J, Mazzali M 2003 Is there a pathogenetic role for uric acid in hypertension and cardiovascular and renal disease? Hypertension 41:1183-1190

3. Krishnan E, Kwoh CK, Schumacher HR, Kuller L 2007 Hyperuricemia and incidence of hypertension among men without metabolic syndrome. Hypertension 49:298-303

4. Mellen PB, Bleyer AJ, Erlinger TP, Evans GW, Nieto FJ, Wagenknecht LE, Wofford MR, Herrington DM 2006 Serum uric acid predicts incident hypertension in a biethnic cohort: the atherosclerosis risk in communities study. Hypertension 48:1037-1042

5. Perlstein TS, Gumieniak O, Williams GH, Sparrow D, Vokonas PS, Gaziano M, Weiss ST, Litonjua AA 2006 Uric acid and the development of hypertension: the normative aging study. Hypertension 48:1031-1036

6. Alper AB Jr, Chen W, Yau L, Srinivasan SR, Berenson GS, Hamm LL 2005 Childhood uric acid predicts adult blood pressure: the Bogalusa Heart Study. Hypertension 45:34-38

7. Feig DI, Johnson RJ 2003 Hyperuricemia in childhood primary hypertension. Hypertension 42:247-252

8. Verdecchia P, Porcellati C, Schillaci G, Borgioni C, Ciucci A, Battistelli M, Guerrieri M, Gatteschi C, Zampi I, Santucci A 1994 Ambulatory blood pressure. An independent predictor of prognosis in essential hypertension. Hypertension 24:793801

9. Clement DL, De Buyzere ML, De Bacquer DA, de Leeuw PW, Duprez DA, Fagard RH, Gheeraert PJ, Missault LH, Braun JJ, Six RO, Van Der Niepen P, O'Brien E 2003 Prognostic value of ambulatory blood-pressure recordings in patients with treated hypertension. N Engl J Med 348:2407-2415

10. Hansen TW, Jeppesen J, Rasmussen S, Ibsen H, Torp-Pedersen C 2005 Ambulatory blood pressure and mortality: a population-based study. Hypertension 45:499-504

11. Sorof JM, Cardwell G, Franco K, Portman RJ 2002 Ambulatory blood pressure and left ventricular mass index in hypertensive children. Hypertension 39:903-908

12. Richey PA, Disessa TG, Hastings MC, Somes GW, Alpert BS, Jones DP 2008 Ambulatory blood pressure and increased left ventricular mass in children at risk for hypertension. J Pediatr 152:343-348

13. 1996 Update on the 1987 task force report on high blood pressure in children and adolescents: a working group report from the national high blood pressure education program. National high blood pressure education program working group on hypertension control in children and adolescents. Pediatrics 98:649-658

14. National high blood pressure education program working group on high blood pressure in children and adolescents 2004. The fourth report on the diagnosis, evaluation, and treatment of high blood pressure in children and adolescents. Pediatrics 114:555-576

15. Richey PA, Jones CL, Harshfield GA, Somes GW, Johnson KC, Bailey JE, Soberman JE 1997 The AM5600 ambulatory blood pressure recording system. Blood Press Monit 2:193-195

16. Kuczmarski RJ, Ogden CL, Grummer-Strawn LM, Flegal KM, Guo SS, Wei R, Mei Z, Curtin LR, Roche AF, Johnson CL 2000 CDC growth charts: United States. Adv Data: $1-27$

17. Soergel M, Kirschstein M, Busch C, Danne T, Gellermann J, Holl R, Krull F, Reichert H, Reusz GS, Rascher W 1997 Oscillometric twenty-four-hour ambulatory blood pressure values in healthy children and adolescents: a multicenter trial including 1141 subjects. J Pediatr 130:178-184 
18. Schwartz GJ, Brion LP, Spitzer A 1987 The use of plasma creatinine concentration for estimating glomerular filtration rate in infants, children, and adolescents. Pediatr Clin North Am 34:571-590

19. Ford ES, Li C, Cook S, Choi HK 2007 Serum concentrations of uric acid and the metabolic syndrome among US children and adolescents. Circulation 115:2526 2532

20. Prebis JW, Gruskin AB, Polinsky MS, Baluarte HJ 1981 Uric acid in childhood essential hypertension. J Pediatr 98:702-707

21. Falkner B, Gidding SS, Ramirez-Garnica G, Wiltrout SA, West D, Rappaport EB 2006 The relationship of body mass index and blood pressure in primary care pediatric patients. J Pediatr 148:195-200

22. Stapleton FB, Linshaw MA, Hassanein K, Gruskin AB 1978 Uric acid excretion in normal children. J Pediatr 92:911-914

23. Goldstein HS, Manowitz P 1993 Relation between serum uric acid and blood pressure in adolescents. Ann Hum Biol 20:423-431

24. Matsuura F, Yamashita S, Nakamura T, Nishida M, Nozaki S, Funahashi T, Matsuzawa Y 1998 Effect of visceral fat accumulation on uric acid metabolism in male obese subjects: visceral fat obesity is linked more closely to overproduction of uric acid than subcutaneous fat obesity. Metabolism 47:929-933
25. Sorof JM, Poffenbarger T, Franco K, Portman R 2001 Evaluation of white coat hypertension in children: importance of the definitions of normal ambulatory blood pressure and the severity of casual hypertension. Am J Hypertens 14:855-860

26. Cannon PJ, Stason WB, Demartini FE, Sommers SC, Laragh JH 1966 Hyperuricemia in primary and renal hypertension. N Engl J Med 275:457-464

27. Klein R, Klein BE, Cornoni JC, Maready J, Cassel JC, Tyroler HA 1973 Serum uric acid. Its relationship to coronary heart disease risk factors and cardiovascular disease, Evans County, Georgia. Arch Intern Med 132:401-410

28. Zoccali C, Maio R, Mallamaci F, Sesti G, Perticone F 2006 Uric acid and endothelial dysfunction in essential hypertension. J Am Soc Nephrol 17:1466-1471

29. Price KL, Sautin YY, Long DA, Zhang L, Miyazaki H, Mu W, Endou H, Johnson RJ 2006 Human vascular smooth muscle cells express a urate transporter. J Am Soc Nephrol 17:1791-1795

30. Mazzali M, Hughes J, Kim YG, Jefferson JA, Kang DH, Gordon KL, Lan HY, Kivlighn S, Johnson RJ 2001 Elevated uric acid increases blood pressure in the rat by a novel crystal-independent mechanism. Hypertension 38:1101-1106

31. Feig DI, Mazzali M, Kang DH, Nakagawa T, Price K, Kannelis J, Johnson RJ 2006 Serum uric acid: a risk factor and a target for treatment? J Am Soc Nephrol 17:S69-S73

32. Lurbe E, Sorof JM, Daniels SR 2004 Clinical and research aspects of ambulatory blood pressure monitoring in children. J Pediatr 144:7-16 